\title{
Técnicas de Mineração de Dados para Investigação da Saúde Mental de Professores: Uma Revisão Sistemática
}

\section{Data Mining Techniques to Investigate Teachers' Mental Health: A Systematic Review}

\author{
Cleonides Silva Dias Gusmão, UFS, cleonides_silva@hotmail.com \\ Renê Pereira de Gusmão, UFS, rene@dcomp.ufs.br \\ Ivan Gomes Ferreira Júnior, UFS, ivan.junior@dcomp.ufs.br
}

\begin{abstract}
Resumo
O magistério é identificado como uma profissão de alto risco, sendo a segunda profissão no ranking mundial de portadores de doenças ocupacionais. Diante disso, este estudo tem como objetivo apresentar uma revisão sistemática da literatura acerca da aplicação de técnicas de mineração de dados para analisar a saúde mental de professores. Para isso, foi realizado um levantamento em 4 bases de artigos científicos com 26 artigos sendo considerados relevantes. Como resultado, a Síndrome de Burnout esteve presente na maioria dos estudos, tendo como principal variável associada as características relacionadas ao ambiente de trabalho dos professores. Por fim, é importante ressaltar a escassez de estudos em relação a saúde mental de professores com a utilização de técnicas de mineração de dados, como é possível observar pela necessidade de reformulação da string de busca e inclusão de mais duas bases de dados para realização dessa pesquisa, tendo em vista o número reduzido de trabalhos encontrados.
\end{abstract}

Descritores: mineração de dados, saúde mental, professores

\begin{abstract}
Teaching is identified as a high-risk profession, being the second profession in the world ranking of people with occupational diseases. Therefore, this study aims to present a systematic review of the literature on the application of data mining techniques to analyze the mental health of teachers. For this, a survey was carried out on 4 scientific databases of articles, in which 26 articles were considered relevant. As a result, Burnout Syndrome was present in most studies, with the main variable associated with the characteristics related to the teachers; work environment. Finally, it is important to highlight the scarcity of studies regarding the mental health of teachers with the use of data mining techniques, as can be seen by the need to reformulate the search string and include two more databases to perform this research, in view of the small number of works found.
\end{abstract}

Keywords: data mining, mental health, teachers 


\section{Introdução}

O magistério é identificado como uma profissão de alto risco, sendo a segunda profissão no ranking mundial de portadores de doenças ocupacionais (Vasconcellos citado por Batista et al., 2010, p.503). No que diz respeito à saúde mental, o estresse chegou ao nível de ser considerado um problema de saúde pública (Lima, 2005, p.1). Quando o estresse no trabalho ocorre diariamente, a síndrome de burnout parece ser uma das nocivas consequências (Maslach, 2003, p. 17). Tal síndrome é composta por três dimensões: a exaustão emocional, a despersonalização e a baixa realização pessoal (Maslach, 1982, p.2).

Torna-se grande a relevância quanto a investigação dessa síndrome, tendo em vista a gravidade das consequências que podem acompanhá-la (Sousa \& Mendonça, 2009, p. 499). Mais especificamente em relação ao magistério, a saúde mental dos professores é algo preocupante, tendo em vista a alta prevalência das dimensões da síndrome de Burnout nessa categoria profissional (p. 506; 509), o que pode impactar no desempenho desses profissionais, agravando a qualidade do trabalho desempenhado por eles e, consequentemente, em prejuízos ao ensino e aprendizagem. Nesse sentido, tornase essencial o estudo voltado a situação de saúde desse profissional para que o mesmo possa ser abarcado no tocante às políticas públicas de saúde (Batista et al., 2010, p.510).

Quando a atenção é direcionada para o entorno dos professores, nota-se uma remuneração baixa associada a ambientes inapropriados para o desempenho da sua função (Tabeleão et al., 2011, p. 2401). Adicionalmente, os profissionais estão com a realidade laboral permeada de sobrecarga de trabalho e avaliam a profissão do magistério como algo estressante (Batista et al., 2010, p.509). Nesse sentido, a presença ininterrupta de tais estressores podem ter como consequência a síndrome de burnout (Leite et al., 2019, p. 171).

Métodos computacionais são cada vez mais utilizados para a análise da saúde mental, como é possível observar no estudo realizado por Alonso et al. (2019, p. 161). Dentro desse contexto, a mineração de dados é um conjunto de técnicas capazes de identificar padrões, relacionamentos e modelos, sendo possível, inclusive, através dela auxiliar no processo de decisão para diagnóstico e plano de tratamento de doenças (Candelieri et al., 2011, p. 272).

Diante do exposto, o presente estudo tem como objetivo apresentar uma revisão bibliográfica da literatura sobre a aplicação das técnicas de mineração de dados para investigação da saúde mental em professores, independente do grau em que o mesmo leciona, incluindo-se artigos direcionados aos professores de ensino pré-escolar, fundamental, médio e universitário. Sendo assim, este artigo está organizado da

seguinte forma: Introdução, Metodologia, Resultados e Discussões e, por fim, Conclusão.

\section{Métodos}

Nesta seção, descrever-se-á como foi desenvolvida a Revisão Sistemática, abarcando a definição dos objetivos da pesquisa, as questões de pesquisa, a string de busca e os critérios de inclusão e exclusão que foram aplicados nos estudos primários. Primeiramente foi definida a string de busca e as bases onde seriam aplicadas. Após a 
aplicação da string de busca nas bases de dados selecionadas, foram obtidos os estudos primários. Em seguida, foi feita a leitura dos títulos e resumos dos estudos obtidos e foram aplicados os critérios de inclusão e exclusão. Por fỉm, a leitura integral dos estudos foi realizada para a extração das principais variáveis e sumarização dos resultados. A seguir, serão apresentados todas essas etapas em detalhes.

\subsection{Questões de Pesquisa}

O objetivo da pesquisa foi identificar a aplicação das técnicas de mineração de dados e estatísticas para investigação de transtornos mentais em professores. Para cumprir com esse objetivo principal, buscou-se responder as seguintes questões:

- Quais os transtornos mentais mais comuns em professores?

- Quais as técnicas de mineração de dados mais utilizadas para investigação dos transtornos mentais em professores?

- Quais os instrumentos mais utilizados para a investigação dos transtornos mentais em professores?

- Quais as variáveis associadas a cada transtorno mental em professores?

- Qual a nacionalidade dos participantes das amostras?

\subsection{String de Busca}

Antes da construção da string de busca, primeiro foi realizada a seleção das palavras chaves utilizadas na pesquisa. As palavras foram utilizadas tanto na língua portuguesa quanto na inglesa e podem ser visualizadas na Tabela 1.

Tabela 1 - Palavras-Chave utilizadas na string de busca

\begin{tabular}{|c|c|}
\hline Palavra Chave & Sinônimos em Inglês \\
\hline Saúde Mental & Mental Health \\
\hline Distúrbio Mental & Mental Disorder \\
\hline Burnout & Burnout \\
\hline Depressão & Depression \\
\hline Estresse & Stress \\
\hline Mineração de Dados & Data Mining \\
\hline Algoritmos & Algorithms \\
\hline Aprendizagem de Máquina & Machine Learning \\
\hline Correlação & Correlation \\
\hline Análise Estatística & Statistical Analysis \\
\hline Regressão & Regression \\
\hline
\end{tabular}

A string de busca foi desenvolvida através da combinação das palavras-chave, sendo apresentada na Tabela 2. Algumas reformulações nas palavras-chave acima e, consequentemente, na string de busca, foram necessárias para se obter um maior número de trabalhos. Acrescentou-se "burnout", "estresse", "correlação", "análise estatística", "regressão" para que a busca fornecesse um maior número de artigos. 
Tabela 2 - String utilizada para realizar as buscas nas bases eletrônicas.

\begin{tabular}{|c|}
\hline String de Busca \\
\hline $\begin{array}{c}\text { (professor) AND (correlation or regression or "statistical analysis" or "data mining" } \\
\text { or algorithm or "machine learning") AND (stress or depression or burnout or "mental } \\
\text { health" or "mental disorder" }\end{array}$ \\
\hline
\end{tabular}

\subsection{Base de Artigos Científicos}

A string de busca foi submetida em quatro bases: Scopus (https://www.scopus.com/), SciELO (Scientific Electronic Library Online) (https://scielo.org/), IEEE Xplore (https://ieeexplore.ieee.org/) e Web of Science (https://clarivate.com/webofsciencegroup/solutions/web-of-science/). Inicialmente, apenas Scielo e IEEE foram as bases de dados incluídas. Porém, com a falta de estudo acerca do tema, fez-se necessário a adição de outras bases. Sendo assim, Web of Science e Scopus foram as bases adicionadas.

Para que não houvesse restrições de download, utilizou-se o portal de periódicos da CAPES (https://www.periodicos.capes.gov.br/) para coleta dos artigos. Durante a execução da busca foram utilizadas as ferramentas de filtragem de cada base, visando considerar na busca somente o título, resumo e palavras-chave dos artigos, sendo considerados artigos publicados no período de 2010 a 2020.

\subsection{Critérios de Seleção}

Os critérios de Exclusão e Inclusão podem ser vistos abaixo. É importante destacar que os artigos selecionados necessitaram apresentar todos os critérios de inclusão.

Os critérios de Exclusão foram:

- Trabalhos que não utilizaram técnicas de mineração de dados ou análise estatística.

- Trabalhos que não investigaram a saúde mental de professores.

Os Critérios de Inclusão foram:

- Trabalhos que investigaram a saúde mental de professores.

- Trabalhos publicados entre os anos de 2010-2020.

- Trabalhos que utilizaram técnicas de mineração de dados ou estatística.

\section{Resultados e Discussões}

Nesta seção são apresentados e discutidos os resultados obtidos. Inicialmente, motrar-se-á os resultados em cada etapa do procedimento de seleção de estudos, em seguida, discutir-se-á os resultados relacionados a cada questão de pesquisa.

É possível notar que na primeira etapa, que consistiu na aplicação da string de busca, 215 trabalhos foram encontrados. Na segunda etapa, que consistiu na leitura do título, resumo e aplicação dos critérios de inclusão e exclusão, sobraram 31 artigos. Na última etapa, que consistiu na leitura integral dos trabalhos, restaram 26 artigos, conforme mostrado na Tabela 3.

A Figura 1 evidencia a distribuição dos trabalhos pelas bases de dados selecionadas durante os últimos 10 anos. A linha no gráfico refere-se ao total de artigos publicados no ano. 
Tabela 3 - Resultados das buscas

\begin{tabular}{|c|c|c|c|}
\hline Base & Etapa 1 & Etapa 2 & Etapa 3 \\
\hline Scielo & 18 & 15 & 5 \\
\hline Scopus & 125 & 82 & 14 \\
\hline Web of Science & 72 & 58 & 7 \\
\hline IEEE & 0 & 0 & 0 \\
\hline Total & 215 & 31 & 26 \\
\hline
\end{tabular}

Nota-se que as publicações começam a ter um aumento em 2017 e caem bruscamente em 2020. Uma das explicações pode ser o período de realização da revisão, que aconteceu nos primeiros 3 meses de 2020, sendo assim inviável a inclusão dos meses restantes do ano, o que inevitavelmente reduziu o total de artigos encontrados em 2020. Um ponto importante é a quantidade de artigos encontrados nas bases da Scopus e Web of Science, já que se mantiveram constantes no período escolhido para este estudo. Por outro lado, o IEEE Xplore apesar de ser um repositório relevante, não possui publicações sobre técnicas e algoritmos de mineração de dados para verificar a saúde mental de professores. Esta ausência de estudos sugere que esse tema necessita de uma maior atenção, como já citado por Vasconcellos (citado por Batista et al., 2010, p.503), o qual chama a atenção para alta incidência de doenças ocupacionais em professores. Dentre as doenças ocupacionais, os profissionais têm se preocupado com a alta ocorrência da síndrome de burnout, a qual pode interferir na qualidade do processo de ensino-aprendizagem (Batista et al., 2010, p. 506; 509, 510).

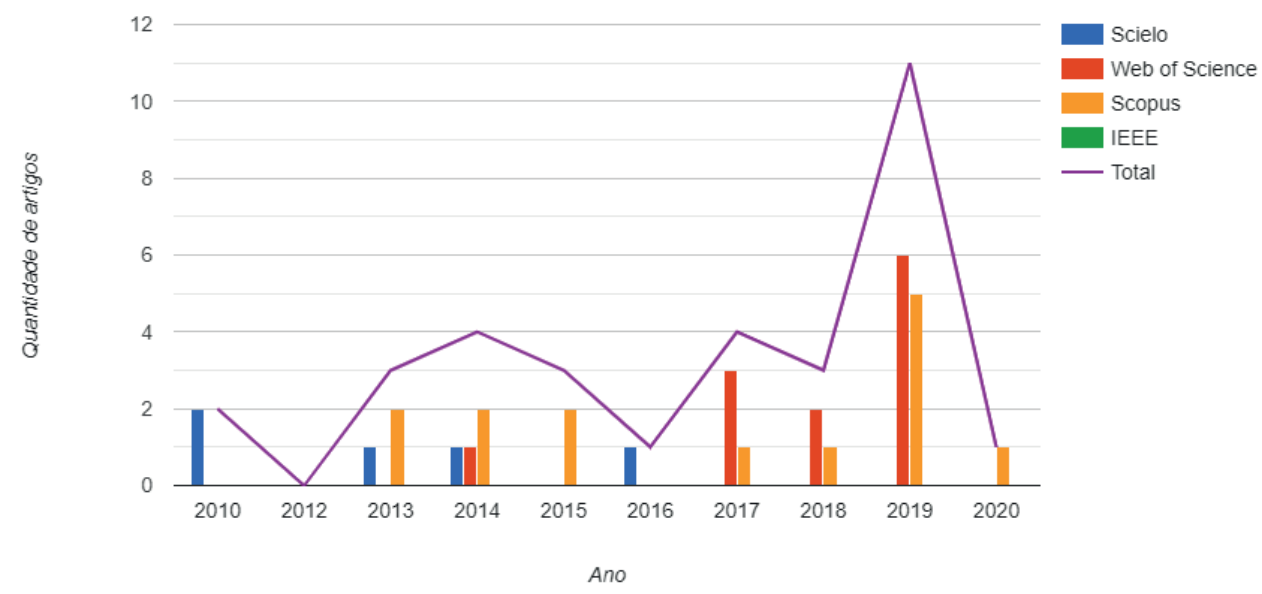

Figura 1 - Artigos por ano em cada base

\subsection{Quais os transtornos mentais mais comuns em professores?}

Em relação aos transtornos encontrados, $65,4 \%$ dos artigos têm a Síndrome de Burnout como transtorno mental foco do estudo. Esse dado é concordante com a alta prevalência dos fatores dessa síndrome nessa categoria profissional (Batista et al., 2010, p. 506; 509). Os outros trabalhos encontrados têm o seu foco direcionado ao estudo do Estresse $(19,2 \%)$ e Estresse ocupacional (15,4\%). Essa variável também está relacionada a síndrome de burnout, como evidenciado por Maslach (2003, p. 17). Apesar disso, é importante destacar que este resultado pode estar enviesado pela string 
de busca que tem "Burnout" e "Estresse" entre suas palavras-chave.

\subsection{Quais as técnicas de mineração de dados mais utilizadas para investigação dos transtornos mentais em professores?}

A mineração de dados auxilia na análise de grandes conjuntos de dados. Através dela importantes informações são extraídas dos dados e, com isso, eles são transformados em conhecimento sistemático (Han, Kamber \& Pei, 2012, p. 1 e p. 2). Na maioria dos artigos analisados nesse estudo foram utilizadas estatísticas para identificar padrões, relacionamentos e modelos voltados a síndrome de Burnout em professores, como propõe a mineração de dados (Candelieri et al., 2011, p. 272).

No geral, percebe-se que as técnicas de mineração de dados e estatística mais utilizadas foram a regressão (41\%) e a correlação (33,3\%) como é mostrado na Figura 2. A correlação é qualquer relação estatística (causal ou não causal) entre duas variáveis. A análise de regressão é similar, porém, enquanto a análise de correlação permite concluir a força da relação entre duas variáveis, a regressão pode descobrir qual efeito de uma variável sobre a outra (Dancey \& Reidy 2006). Este resultado também pode estar enviesado pela string de busca. Vale ressaltar que as variações da regressão e correlação foram agrupadas para facilitar na visualização do gráfico.

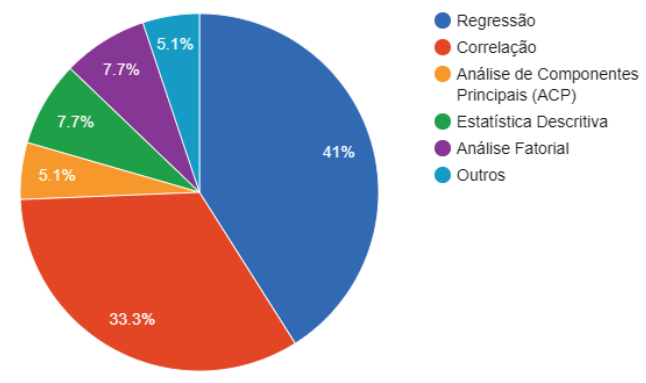

Figura 2 - Porcentagem geral de algoritmos e técnicas utilizados

Analisando-se cada transtorno individualmente, as regressões aparecem em $44,4 \%$ dos estudos que têm seu foco direcionado ao estudo da Síndrome de Burnout, como é apresentado na Figura 3. Dentre as regressões, temos nesse estudo a regressão linear, regressão linear múltipla, regressão logística, regressão quadrática, entre outras. Nota-se na Figura 4 que as regressões também foram mais utilizadas quando comparadas a correlação, no que se trata da investigação do estresse ocupacional, sendo $38,5 \%$ e $30,8 \%$, respectivamente.

\subsection{Quais os instrumentos mais utilizados para a investigação dos transtornos mentais em professores?}

Em relação aos instrumentos utilizados para investigação da Síndrome de Burnout, percebe-se que o Maslach Burnout Inventory (MBI) e suas variantes foram os mais utilizados.O MBI foi projetado para medir burnout em várias ocupações (Maslach \& Jackson, 1981). Também foram utilizados o Questionário de pressão de publicação validada e Questionário de Burnout no professor (CBP-R) (Moreno-Jimenez et al., 2000) e QPW-5 (Questionnaire on Quality of Work life in Human Service 
Organizations) (Romero-Caraballo, 2019).

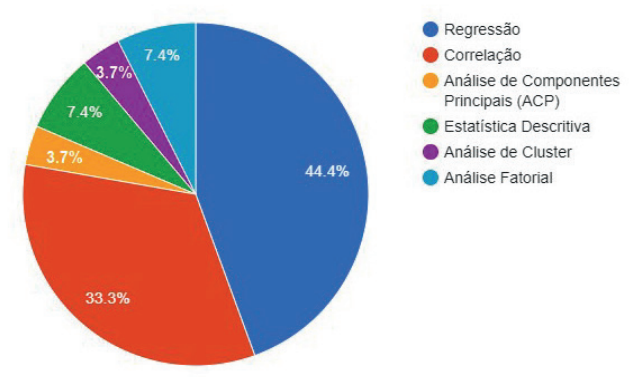

Figura 3 - Porcentagem de algoritmos e técnicas utilizados para burnout

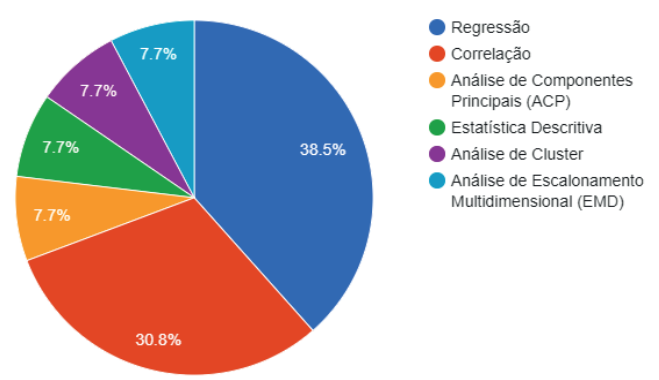

Figura 4 - Porcentagem de algoritmos e técnicas utilizados para estresse

Já para o estresse foram utilizadas a Escala de Estresse Percebido (PSS) (Cohen et al., 1983), Coping Job Scale (Latack 1986), Escala de estresse no trabalho (ETT) (Paschoal \& Tamayo, 2005) e inventário de perfis de valores organizacionais (IPVO) (Álvaro, 2007).

\subsection{Quais as variáveis associadas a cada transtorno?}

No que diz a respeito as variáveis associadas à Síndrome de Burnout, destacamse as condições organizacionais (Cárdenas Rodríguez et al., 2014, p.18), o que, segundo o mesmo autor, acaba por interferir no desempenho desse profissional. Os problemas sociais e com os alunos (Sánchez et al., 2019, p. 121) também foram destacados como fatores associados ao burnout. Além dessas variáveis, também foram encontradas associações com: coping (García-Arroyo \& Osca, 2017), nível de cortisol (FajardoZapata et al., 2013), sexo, idade, estado civil, anos de ensino (Flores \& Trujillo, 2018), autonomia, conflito de papel e conflito interpessoais (Carlotto, 2017).

Já para o estresse, as variáveis associadas foram as estratégias de coping, o tempo de carreira (mais de 10 anos), e o gênero (masculino) (Capelo, 2016). Sousa et al. (2018) trazem as variáveis relacionadas a baixa qualidade do sono. Outras variáveis foram: recursos fornecidos pelas instituições e características sociodemográficas (Davydova \& Mikhaylova, 2014), remuneração e compensação, condições de trabalho (Moreira et al., 2010).

\subsection{Qual a nacionalidade dos participantes das amostras?}


Como é apresentado na figura 5, a amostra é predominantemente do Brasil com 8 artigos (cerca de 29,62\%). No Brasil a docência está envolta por inúmeros fatores que contribuem para a não satisfação dos profissionais dessa área, tais como a agregação de tarefas administrativas (Mancebo, 2007). No caso de professores universitários, Mancebo (2007, p. 4) afirma: "Ele agora é responsável não apenas pela sala de aula e pelo desenvolvimento de sua pesquisa, mas por um crescente número de tarefas, como o preenchimento de inúmeros relatórios e formulários, a emissão de pareceres, a captação de recursos para viabilizar seu trabalho e até para o bom funcionamento da universidade".

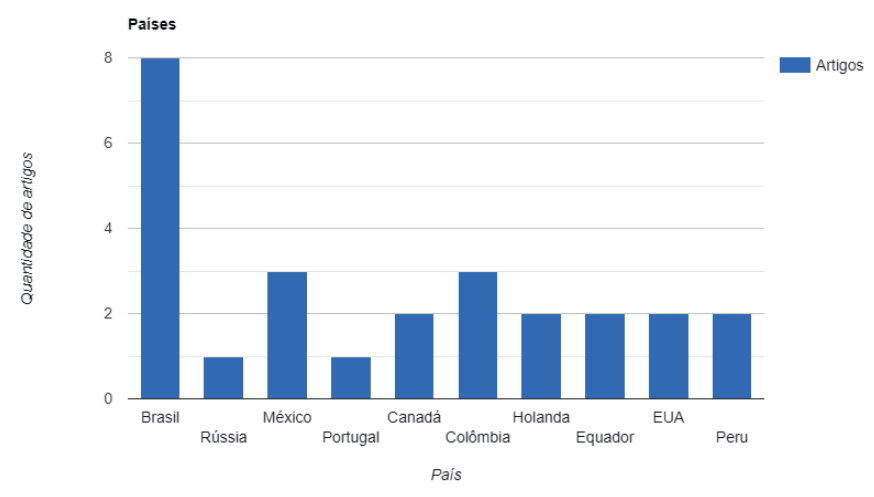

Figura 5 - Nacionalidade das amostras

\section{Conclusões}

Este trabalho teve como objetivo identificar estudos na literatura acadêmica que abordaram técnicas de mineração de dados e estatísticas para averiguar a saúde mental de professores. A revisão sistemática foi realizada com artigos publicados no período de 2010 a março de 2020. As bases de dados consultadas para construção dessa revisão foram a Scielo, Scopus, IEEE Xplore e Web of Science.

A busca inicial forneceu 215 estudos, que após a aplicação de critérios de inclusão e exclusão foram reduzidos para 31 artigos dos quais se procedeu uma leitura completa. Por fim, restaram 26 estudos que foram incluídos na revisão sistemática, dos quais foram extraídas informações das variáveis selecionadas.

Através desses resultados, pode-se destacar a redução considerável de artigos após a aplicação dos critérios de inclusão e exclusão. Essa decrescida substancial é contraditória quando nos voltamos para a relevância do tema.

Por meio dessa revisão de literatura também foi possível constatar a importância de se voltar ao estudo da síndrome de burnout em relação aos profissionais docentes, especialmente no tocante ao seu ambiente de trabalho que destacou-se como uma importante variável associada. No Brasil, a precarização da educação é uma realidade constante, e essa área tem passado por cortes de investimento consistentes e frequentes. $\mathrm{O}$ professor e seu ambiente de trabalho não são imunes a tais acontecimentos. Devido a essa realidade, torna-se ainda mais importante o estudo voltado a saúde mental desses profissionais.

Uma limitação a ser destacada nessa pesquisa é a string de busca, a qual teve de ser reajustada algumas vezes, acrescentado-se algumas palavras-chave, para que 
pudesse ser obtido um maior número de trabalhos, tendo em vista a modesta quantidade de artigos encontrados. Inicialmente, a string de busca voltava-se apenas aos professores universitários e excluía trabalhos que utilizavam estatística para análise de dados.

Como trabalhos futuros, propõe-se a análise comparativa dos algoritmos de mineração de dados para avaliar a Síndrome de Burnout em professores. Além disso, estudos acerca da estratificação dos professores por nível de ensino também é interessante, tendo o intuito de analisar e prever a síndrome de burnout em professores do ensino básico, fundamental e superior.

\section{Referências Bibliográficas}

ALONSO, S.G.; TORRE-DÍEZ, I.; HAMRIOUI, S.; LÓPEZ-CORONADO, M.; BARRENO, D.C.; NOZALEDA, L.M.; FRANCO, M. Data mining algorithms and techniques in mental health: a systematic review. Journal of medical systems, v. 42, n.161, p. 1-15, 2019.

ÁLVARO, T. Impacto dos Valores da Organização sobre o Estresse Ocupacional. RAC - Eletrônica, v. 1, n. 2, p. 20-33, 2007.

BATISTA, J.B.V.; CARLOTTO, M.S.; COUTINHO, A.S.; AUGUSTO, L.G.S. Prevalência da Síndrome de Burnout e fatores sociodemográficos e laborais em professores de escolas municipais da cidade de João Pessoa, PB. Revista Brasileira de Epidemiologia, São Paulo, v. 13, n. 3, p. 502-512, 2010.

CANDELIERI, A.; DOLCE, G.; RIGANELLO, F.; SANNITA, W.G. Data Mining in Neurology. In: Funatsu, K (Ed). Knowledge-Oriented Applications in Data Mining. Rijeka. Croatia: InTech, 2011. p. 261-276.

CAPELO, R.; POCINHO, M. Estratégias de coping: contributos para a diminuição do estresse docente. Psicologia, Saúde \& Doenças, Lisboa. v. 17, n. 2, p. 282-294, 2016.

RODRÍGUEZ, M.C.; HINOJOSA, L.M.M.; RAMÍREZ, M.T.G. Evaluación del desempeño docente, estrés y burnout en profesores universitarios. Actualidades Investigativas en Educación, San José. v. 14, n. 1, p. 93-114, 2014.

CARLOTTO, M.S.; CÂMARA, S.G. Riscos psicossociais associados à síndrome de burnout em professores universitários. Avances en Psicología Latinoamericana, Colombia. v.35, n. 3, p. 447-457, 2017.

COHEN, S.; KAMARCK, T.; MERMELSTEIN, R. A global measure of perceived stress. Journal of Health and Social Behavior. v. 24, n. 4, p. 385-396, 1983.

DANCEY, C.P.; REIDY, J. Estatística sem Matemática para Psicologia: Usando SPSS para Windows. Porto Alegre: Artmed. 2006.

DAVYDOVA, I.; MIKHAYLOVA, Y. Occupational Stress and Job Satisfaction among Professors of Russian Higher Educational Institutions. Voprosy obrazovaniya / Educational Studies Moscow. n. 4., p. 169-183, 2014.

FAJARDO-ZAPATA, A.; MONTEJO-ANGEL, F.; MOLANO-VARGAS， G.; HERNANDEZ-NIÑO, J.; QUINTERO-GUZMAN, A. Correlación entre los Factores de Riesgo Intralaboral y los Niveles Séricos de Cortisol en Docentes Universitarios. Ciencia \& trabajo, Santiago. v. 15, n. 46, p. 1-6, 2013.

GARCÍA-ARROYO, J.A.; OSCA, A. Coping with burnout: analysis of linear, nonlinear and interaction relationships. Anales de Psicología. v.33, n.3, p.722-731, 2017. 
LATACK, J.C. Coping with job stress: measures and future directions for scale development. Journal of applied psychology. v. 71, n.3, p. 377-385, 1986.

LEITE. T.; FERNANDES, J.; ARAUJO, F.; PEREIRA, X.; AZEVEDO, D.; LUCENA, E. Prevalência e fatores associados da síndrome de Burnout em docentes universitários.

Revista Brasileira de Medicina do Trabalho. v.17, n.2, p.170-179, 2019.

LIMA, F.V. Correlação entre variáveis preditoras de estresse e o nível de estresse. Brasília: Universidade Católica de Brasília, 2005. 147p. Dissertação de Mestrado.

MANCEBO, D. Trabalho docente: subjetividade, sobreimplicação e prazer. Psicologia: Reflexão e Crítica. Porto Alegre. v. 20, n. 1, p. 74-80, 2007.

MASLACH, C. Burnout: the cost of caring. Englewood Cliffs: Prentice - Hall, 1982.

MASLACH C. Burnout: the Cost of Caring. Cambridge: Malor Books, 2003.

MASLACH, C.; JACKSON, S. The Measurement of Experienced Burnout. Journal of Organizational Behavior. v.2, n.2, p. $99-113,1981$.

MOREIRA, H.R.; NASCIMENTO, J.V.; SONOO, C.N.; BOTH, J. Qualidade de vida do trabalhador docente em educação física do estado do Paraná, Brasil. Revista Brasileira de Cineantropometria \& Desempenho Humano, Florianópolis. v. 12, n. 6, p. 435-442, 2010.

MORENO-JIMENEZ, B.; GARROSA, E.; GONZÁLEZ, J. La evaluación del estrés y el burnout del profesorado: El CBP-R. Revista de Psicología del Trabajo y de las Organizaciones. v. 16, n. 2, p. 151-171, 2000.

PASCHOAL, T.; TAMAYO, A. Impacto dos valores laborais e da interferência família: trabalho no estresse ocupacional. Psicologia: Teoria e Pesquisa, Brasília. v. 21, n. 2, p. 173-180, 2005.

FLORES, E.A.R.; TRUJILLO, M.A.S. Síndrome de Burnout y variables sociodemográficas en docentes de una universidad privada de Lima. Revista de Investigación Educativa. v. 36, n. 2, p. 401-419, 2018.

ROMERO-CARABALLO, M.P. Working conditions and the meaning of the academic profession in Colombian university professors. Acta Colombiana de Psicología. v. 12, n.22, p. $267-291,2019$.

SANCHEZ, D.V.; GARCÍA, A.J.; CORZO, I.G; MORENO, M.O. Factores psicosociales y síndrome de Burnout en académicos de una universidad pública de México. Propósitos y Representaciones, Lima, v. 7, n. 3, p. 111-126, 2019.

SOUSA, I. F.; MENDONÇA, H. Burnout em professores universitários: impacto de percepções de justiça e comprometimento afetivo. Psicologia: Teoria e Pesquisa, v. 25, n. 4, p. 499-508, 2009.

SOUSA, A.; SANTOS, R.; SILVA, R.M.; SANTOS, C.; LOPES, V.; MUSSI, F. Occupational stress and sleep quality in professors of the health area. Revista da Rede de Enfermagem do Nordeste. v. 19, p. 1 - 8, 2018.

TABELEÃO, V.P.; TOMASI, E.; NEVES, S.F. Qualidade de vida e esgotamento profissional entre docentes da rede pública de Ensino Médio e Fundamental no Sul do Brasil. Cadernos de Saúde Pública, Rio de Janeiro. v. 27, n. 12, p. 2401-2408, 2011. HAN, J.; KAMBER, M.; PEI, J. Data Mining: Concepts and Techniques, $3^{\circ}$ edition, Morgan Kaufmann, 2012. 